\title{
Rotenone-induced energy stress decompensated in ventral mesocerebrum is associated with Parkinsonism progression in rats
}

\author{
QUNHUA BAI $^{1-3}$, JUNLIN HE ${ }^{1}$, YONG TANG ${ }^{4}$, SHIBO WANG $^{5}$, JINGFU QIU ${ }^{1}$, YANG WANG ${ }^{1}$ and CHAO YU $^{2}$ \\ ${ }^{1}$ School of Public Health and Management; ${ }^{2}$ Institute of Life Science, Chongqing Medical University; \\ ${ }^{3}$ Research Center for Medicine and Social Development; ${ }^{4}$ Department of Spinal Cord Injury, \\ Chongqing Orthopedics Hospital of Traditional Chinese Medicine, Chongqing 400016; \\ ${ }^{5}$ Department of Infection Management, The Second Hospital of Hebei Medical University, \\ Shijiazhuang, Hebei 050000, P.R. China
}

Received March 29, 2015; Accepted April 29, 2016

DOI: $10.3892 /$ etm.2016.3352

\begin{abstract}
Parkinson's disease (PD) is the second most common neurodegenerative disorder, which is characterized by the hallmark feature of loss of dopaminergic neurons in the substantia nigra. Energy metabolic disorder is associated with the pathogenesis of PD; however, the development of this disorder is yet to be elucidated. PD-like characteristics have been demonstrated in a rotenone rat model. In the present study, energy metabolism status was investigated in a rat model following intraperitoneal treatment with $1.0 \mathrm{mg} / \mathrm{kg}$ rotenone every $48 \mathrm{~h}$. The behavior and tyrosine hydroxylase-positive levels in the substantia nigra of rats that were treated with rotenone for 24 weeks demonstrated that these rats developed more severe parkinsonism, as compared with that were treated for 16 weeks. Detection of ATP, lactic acid, NADH dehydrogenase $1 \mathrm{mRNA}$ and lactate dehydrogenase B mRNA levels in the ventral mesocerebrum (VM) and skeletal muscle (SM) of the rats that had been treated with rotenone for 16 and 24 weeks demonstrated that the energy stress induced by rotenone progressed in both VM and SM. Notably, the energy stress detected in VM was more severe, and this energy stress was decompensated in the VM of rats that had been treated with rotenone for 24 weeks. The progression of energy stress and the incidence of energy decompensation in VM may be important for the improvement of PD pathology.
\end{abstract}

Correspondence to: Professor Chao Yu, Institute of Life Science, Chongqing Medical University, 1 Yi Xue Yuan Road, Chongqing 400016, P.R. China

E-mail: yuchaom@163.com

Abbreviations: TH, tyrosine hydroxylase; PD, Parkinson's Disease; DA, dopamine; ND1, mitochondrial complex I subunit $\mathrm{ND1}$; LDHB, lactate dehydrogenase $\mathrm{B} ; 16 \mathrm{Wr}$, the rats had rotenone medicated for 16 weeks; $24 \mathrm{Wr}$, the rats had rotenone medicated for 24 weeks; SM, skeletal muscle; VM, ventral mesocerebrum

Key words: rat, rotenone, energy stress, Parkinson disease, ventral mesocerebrum

\section{Introduction}

Parkinson's disease (PD) is characterized by the loss of dopaminergic (DA) neurons in the substantia nigra. PD is the most common neurodegenerative movement disorder, affecting $>1 \%$ of the population that are aged $>60$ (1). Current treatments, such as pharmaceutical agents or deep brain stimulation predominantly target the symptoms and are unable to attenuate or reverse the progression of this disease (2). Understanding $\mathrm{PD}$ at a cellular and molecular level is important to determine novel targets and develop neuroprotective and disease-modifying therapeutic strategies. Although the causes and potential factors remain unknown, environmental factors may have a critical role, such as herbicides or pesticides, organic solvents, carbon monoxide and carbon disulfide $(3,4)$. Notably, various studies have suggested that PD is associated with energy metabolism defects (5-7).

Due to their structure, physiologic activities and high oxidative level of DA metabolism, DA cells in the substantia nigra are energy demanding and prone to energy stress (8). Furthermore, these cells have a relative low capacity for glycolysis compensation and lower glutathione levels $(9,10)$, which ensures that they cannot respond appropriately to energy stress and are thus sensitive to energy disturbances.

Rotenone is a common insecticide and mitochondria complex 1 inhibitor, which is used to induce chronic rotenone rat models with $\mathrm{PD}$-associated features that manifest as DA cells are selectively damaged (11). However, how energy stress is induced and associated with the development of PD-associated in this model remains unknown.

In the present study, rats were treated with rotenone and energy stress status was subsequently detected in ventral mesocerebrum (VM) and skeletal muscle (SM) tissues at two time points following the development of different levels of parkinsonism. The present study aimed to clarify how energy metabolism and compensatory status are associated with the development of PD-associated features and manifestations in rat models. These findings may aid elucidation of how energy metabolism defects are linked to the progression of PD, and whether prevention of the energy stress is vital to attenuating the progression of PD. 


\section{Materials and methods}

Reagents and materials. Rotenone; (Sigma-Aldrich, St. Louis, MO, USA); ATP assay kit (S0026; Beyotime Institute of Biotechnology, Jiangsu, China); lactic acid assay kit (K627-100; BioVision Company, Palo Alto, CA, USA; rabbit anti-rat tyrosine hydroxylase $(\mathrm{TH})$ primary antibody (AB152) and fluorescein isothiocyanate (FITC)-labeled anti-rabbit secondary antibody (AP132F; EMD Millipore, Billerica, MA, USA); TRIzol RNA extraction reagent, RNA reverse transcription reagent and PCR expansion reagent kit (Takara Biotechnology Co., Ltd., Dalian, China); SYBR Green mix (Promega Corporation, Madison, WI, USA); and NADH dehydrogenase 1 (ND1) and lactate dehydrogenase B (LDHB) primers (Shanghai Biotechnology Co., Ltd., Shanghai, China).

Animals and rotenone treatment. Male Wistar rats $(n=40)$, aged 20 months and weighing 554 $\pm 9 \mathrm{~g}$, were obtained from the Third Military Medical University (Chongqing, China). Rats were individually housed and maintained in a temperature- $\left(22 \pm 1^{\circ} \mathrm{C}\right)$ and light-controlled (12-h light/dark cycle) environment with ad libitum access to food and water. The present study was approved by the Animal Care and Use Committee of Chongqing Medical University.

Rats were randomly divided into rotenone $(n=25)$ and control groups $(n=15)$. Rotenone was ultrasonically dissolved in sunflower oil at a concentration of $5 \mathrm{mg} \cdot \mathrm{l}^{-1}$. Rats in the rotenone group were administered $1.0 \mathrm{mg} \cdot \mathrm{kg}^{-1}$ rotenone (i.p.) every $48 \mathrm{~h}$; whereas the control group were treated with the the same volume of sunflower oil solvent. Weights were measured prior to each administration.

Rota-rod test. Motor ability was examined using a rota-rod test system (57602V; Ugo Basile, Chicago, IL, USA) of which the rotation speed was accelerated from $3 \mathrm{rpm}$ to $40 \mathrm{rpm}$ with an acceleration of 0.3 rounds/sec in a reverse direction. Prior to the rota-rod test, rats were trained 10 times every $48 \mathrm{~h}$ following rotenone treatment for 2 weeks. The duration that each rat remained on the bar before falling off was recorded, and this was repeated 6 times for each test.

Tissue preparation. For immunochemical detection, rats were anesthetized by intraperitoneal injection of $30 \mathrm{mg} / \mathrm{kg}$ pentobarbital sodium into the right lower quadrant, and were subsequently transcardially perfused with $4 \%$ paraformaldehyde. Rat brains were harvested and maintained in $4 \%$ paraformaldehyde for $24 \mathrm{~h}$ prior to incubation with $30 \%$ sucrose until equilibrium and storage at $-80^{\circ} \mathrm{C}$ until use.

The rats used for ATP, lactic acid, ND1 mRNA, and LDHB mRNA level detection in the SM and VM were anesthetized by pentobarbital, then the hind leg inside SM and VM part were taken on ice and immediately stored in liquid nitrogen until use. The muscle tissue used for electron microscope were swiftly taken and dissected about $1 \mathrm{~mm}^{3}$ of the volume, stored in glutaral fixative solution until use.

Immunofluorescence detection. Sections of mesocerebrum (60 $\mu$ m-thick) were cut from the rat heart using a frozen sliding microtome. Following exposure to room temperature for $40 \mathrm{~min}$, the sections were stored at $-80^{\circ} \mathrm{C}$ until use.
For immunofluorescent detection of $\mathrm{TH}$, the sections were exposed to room temperature for $1 \mathrm{~h}$, washed in PBS for $10 \mathrm{~min}$, blocked for $1 \mathrm{~h}$ in $5 \%$ bovine serum albumin supplemented with $0.3 \%$ Triton $\mathrm{X}-100$, and incubated with anti-TH primary antibody $(1: 2,000)$ for $72 \mathrm{~h}$ at $4^{\circ} \mathrm{C}$ and $2 \mathrm{~h}$ at room temperature. Following washing three times in PBS for $10 \mathrm{~min}$, the sections were incubated at $37^{\circ} \mathrm{C}$ for $1 \mathrm{~h}$ with FITC-labeled goat anti-rabbit secondary antibody (1:100). Sections were subsequently washed three times for $5 \mathrm{~min}$ in PBS and observed under a fluorescence-microscope (CKX41-32FL; Olympus Corporation, Tokyo, Japan).

Semi-fluorescent quantification of TH-positive cells in the substantia nigra. VM coronal sections were immunofluorescently stained against $\mathrm{TH}$, as described. The number of TH-positive cells in the substantia nigra, including the substantia nigra pars compacta and reticulata, were subsequently analyzed in five continuous sections/rat of the same coronal level of the brain. The respective means of the total cells in one cerebral hemisphere were compared. An Image-Pro Insight 8.0 image analysis system (Media Cybernetics, Inc., Rockville, MD, USA) was used.

ATP and lactic acid detection. Tissues were ground into a fine powder in liquid nitrogen prior to ATP and lactic acid detection, which was performed according to the manufacturer's protocol using a LMax II Luminometer (Molecular Devices LLC, Sunnyvale, CA, USA) and Sunrise remote microplate reader (Tecan Trading AG, Grodlg, Austria).

Electron microscopic observation of muscle mitochondria morphous. SM tissue from the inside of the hind leg was fixed in glutaraldehyde and cut into 50-nm ultrathin sections prior to observation under a transmission electron microscope (Hitachi-7500; Hitachi, Ltd., Tokyo, Japan).

Reverse transcription-quantitative polymerase chain reaction (RT-qPCR).ND1 and LDHB mRNA expression level detection. Following storage at $-80^{\circ} \mathrm{C}$, the tissues were ground into a fine powder in liquid nitrogen and total RNA was isolated from $50 \mathrm{mg}$ tissue using $1 \mathrm{ml}$ cold TRIzol reagent, chloroform, isopropanol and 70\% ethanol. Subsequently, $500 \mathrm{ng}$ RNA was reverse transcribed into cDNA using an RT reagent kit, according to the manufacturer's protocol. cDNA were stored at $-80^{\circ} \mathrm{C}$ until RT-qPCR with the following primers: ND1, sense 5'-AAG CGGCTCCT T CTCCCT ACA AAT-3' and antisense, 5'-GCTCGATTTGTTTCTGCGAGGGTT-3' (119-bp product); $\beta$-actin, sense 5'-AGGCCAACCGTGAAA AGATG-3' and antisense 5'-ACCAGAGGCATACAGGGA CAA-3' (88-bp product); LDHB, sense 5'-GACCTCATCGAA TCCATGCT-3' and antisense 5'-TCGTCCTTCADCTTC TGGTT-3' (161-bp product). The reaction system contained $5 \mu \mathrm{l}$ SYBR Green mix, $30 \mu \mathrm{M}$ sense and antisense primers, $1 \mu \mathrm{l}$ cDNA and RNA-free $\mathrm{H}_{2} \mathrm{O}$ to $10 \mu \mathrm{l}$ and was performed on a Chromo 4 real-time PCR system (Bio-Rad Laboratories, Inc., Hercules, CA, USA). Thermal cycling was performed as follows: $3 \mathrm{~min}$ at $95^{\circ} \mathrm{C}$, followed by 35 cycles of $10 \mathrm{sec}$ at $95^{\circ} \mathrm{C}$ and $20 \mathrm{sec}$ at $55^{\circ} \mathrm{C}$, and $10 \mathrm{sec}$ at $72^{\circ} \mathrm{C}$ and $65^{\circ} \mathrm{C}-95^{\circ} \mathrm{C}$ for the dissociation curve. ND1 and LDHB mRNA expression levels were normalized to $\beta$-actin mRNA levels as the endogenous 
reference, according to the $2^{-\Delta \Delta \mathrm{Cq}}$ method (12). All primer sets exhibited PCR efficiencies similar to the $\beta$-actin primer set $(85-100 \%)$, as determined by the analysis of $\mathrm{Ct}$ across serial dilutions. Negative control reactions without the cDNA template consistently lacked a product. All samples were run in duplicate.

Statistical analysis. Statistical analyses were performed using SPSS 10.0 software (SPSS, Inc., Chicago, IL, USA). Data were expressed as the mean \pm standard deviation of 3-6 independent experiments. Analysis of variance and Student's t-test were performed to determine statistical significance. $\mathrm{P}<0.05$ was considered to indicate a statistically significant difference.

\section{Results}

Rotenone treatment induces behavioral deficits and mortality. Behavioral analysis of the rotenone group demonstrated that the rats were very weak and exhibited difficulty moving and eating when treated with rotenone for 24 weeks; therefore, the 24th week was designated as the endpoint of the present study. Within the first 2 weeks, $32 \%$ of rats $(8 / 25)$ in the rotenone group developed behavioral deficits in response to the treatment, including weight loss, and decreased motor ability and food intake. These behaviors were typically relieved within $48 \mathrm{~h}$. Two of the most responsive rats succumbed to these symptoms in the 2nd week. Although these behavioral deficits were relieved during weeks 4 and 5, they increased in severity and number from the 12th week. During the 24th week, two rats died after one treatment. The control group incurred no fatalities during the present study. These results suggested that the rats were progressively injured by rotenone treatment.

Weight and motor ability varies with rotenone treatment. The respective weights of each rat were measured prior to treatment and each every week, as presented in Fig. 1. The mean weight of rats in the control group increased throughout the research period; whereas rats in the rotenone group exhibited weight loss in the first 4 weeks, followed by a weight increase until the 12th week and a gradual decrease between weeks 16-24. Rats in the rotenone rat model exhibited obvious weight loss, which was regarded to be associated with the damage to gastrointestinal neurons $(13,14)$, which impede digestion. This symptom of weight loss also manifests in patients with PD $(15,16)$. Weight loss was demonstrated in the first 4 weeks in the rotenone group, which is consistent with the results published by Cannon et al (17), and may be caused by the rats' acute inadaptation to the rotenone treatment.

In the present study, a rota-rod test system was used to evaluate how the rats' motor ability was affected by rotenene. Since rotenone treatment made some rats very weak and unable to stay on the bar until 24 to $48 \mathrm{~h}$ later, the rota-rod tests were performed $48 \mathrm{~h}$ post-treatment, once every 4 weeks. The results demonstrated that, in control group, the time that rats remained on the bar initially increased between weeks $4-12,(45.0 \pm 21.4,63.0 \pm 25.8$ and $85.1 \pm 31.5 \mathrm{sec}$, respectively), then was maintained at $91.4 \pm 30.7 \mathrm{sec}$ for weeks $16-24$ (Fig. 2A). For rats in the rotenone group, the time on the bar also increased initially during weeks 4-12 (25.2 \pm 19.2 ,

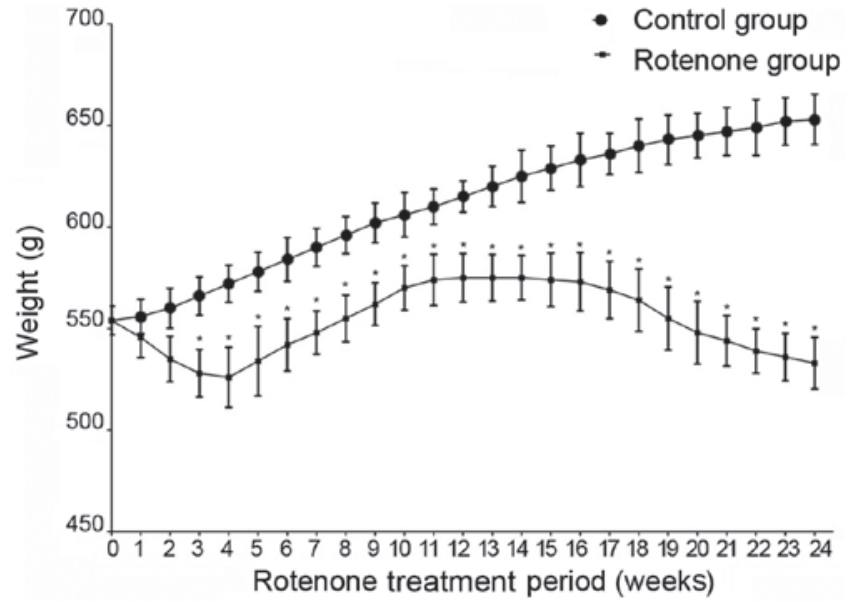

Figure 1. Variation in the weights of the rats during the rotenone treatment period. Data are presented as the mean \pm standard deviation. ${ }^{*} \mathrm{P}<0.05$ vs. the control group.
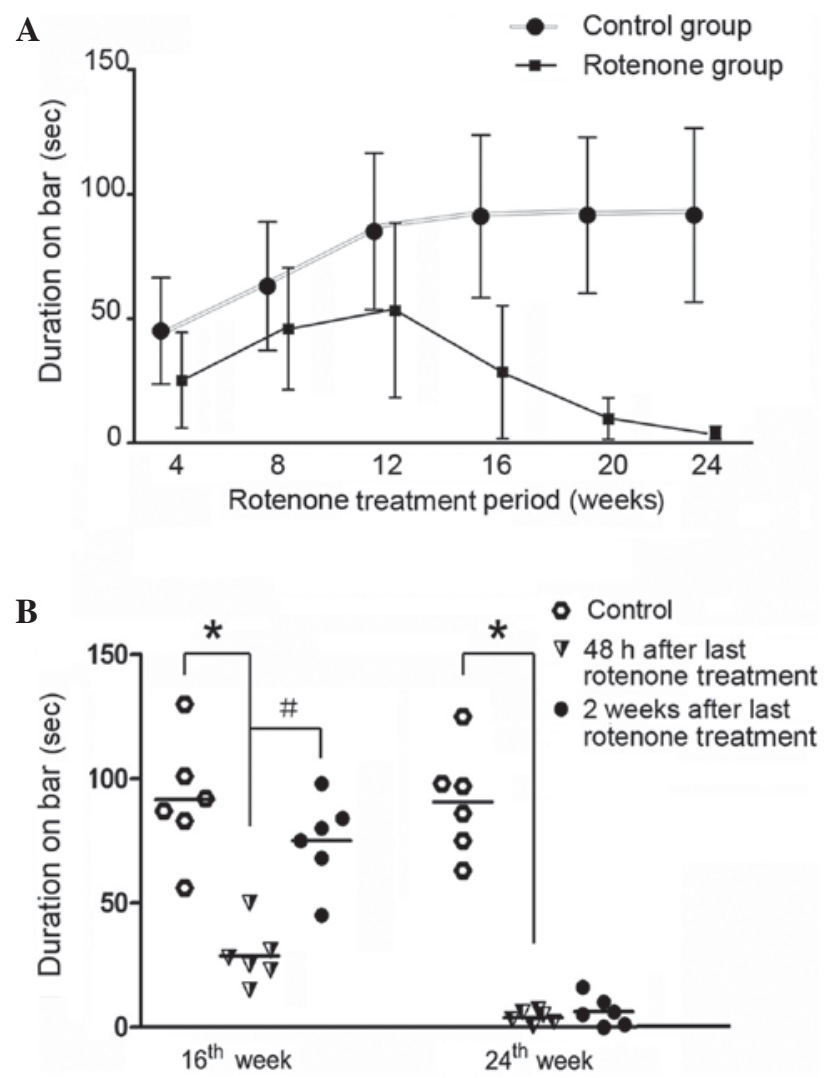

Figure 2. Motor ability of the rats, as determined by rota-rod testing, (A) during the rotenone treatment period ( $\mathrm{n} \geq 12$ rats/group) and (B) $48 \mathrm{~h}$ and 2 weeks after the last rotenone treatment in rats that had been treated for 16 and 24 weeks, respectively ( $n=6$ rats/group). Data are presented as the mean \pm standard deviation. ${ }^{*} \mathrm{P}<0.05$ vs. the control group; ${ }^{\#} \mathrm{P}<0.05$ vs. $48 \mathrm{~h}$ after treatment.

$45.9 \pm 24.5$ and $53.3 \pm 35.1 \mathrm{sec}$, respectively), although at a lower level as compared with the control group, then the time sharply decreased between weeks 16-20 (28.5 $26.7,9.8 \pm 8.3$ and $4.1 \pm 2.6 \mathrm{sec}$, respectively).

In order to elucidate how rotenone treatment affected the motor ability of rats, rota-rod testing was performed 2 weeks 
A
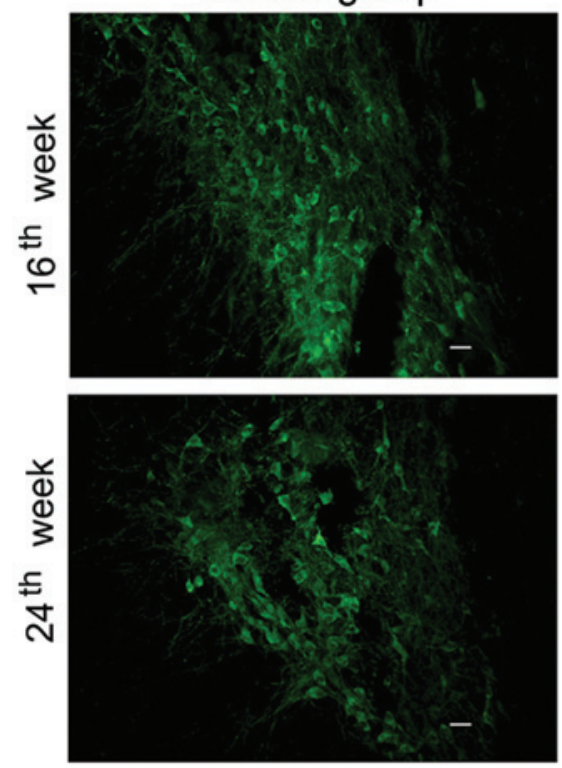

Rotenone group
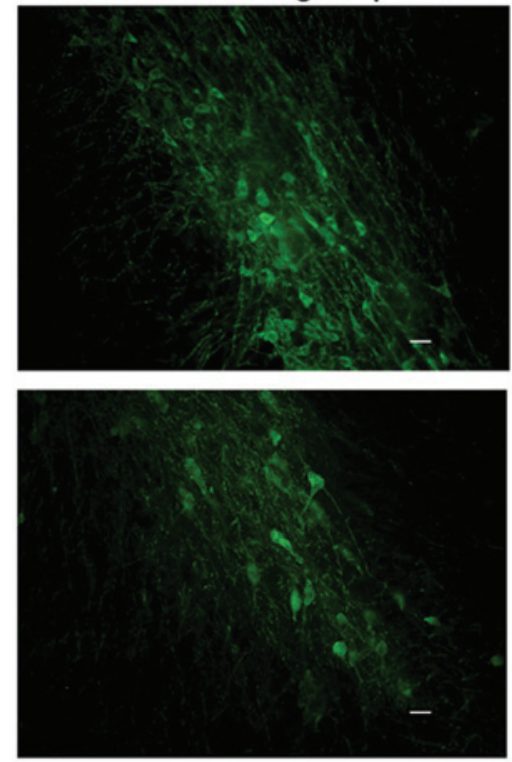

Control group Rotenone group

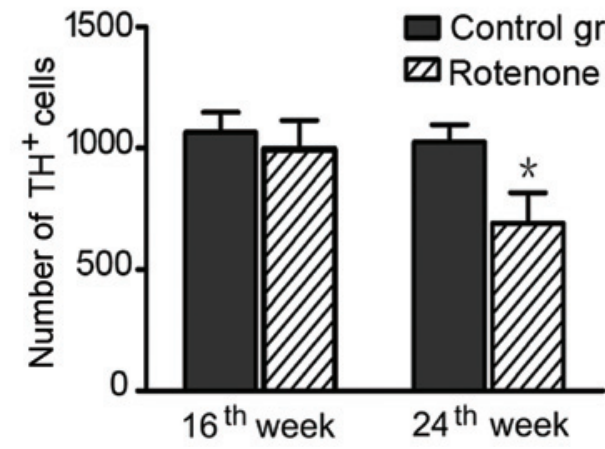

Figure 3. (A) Immunofluorescence detection of TH protein levels in the substantia nigra of control rats and rats administered their final rotenone treatment at weeks 16 and 24, respectively. (scale bar, $20 \mu \mathrm{m}$ ). (B) Semi-fluorescence quantification of TH-positive cells in the substantia nigra of five continuous sections per rat $(n=3)$. Data are presented as the mean \pm standard deviation. ${ }^{*} \mathrm{P}<0.05$ vs. the control group. TH, tyrosine hydroxylase.

after rotenone treatment was terminated for rats treated with rotenone for 16 weeks $(16 \mathrm{Wr})$ and 24 weeks $(24 \mathrm{Wr})$, respectively. The results demonstrated that, 2 weeks after the final rotenone treatment, the duration that rats in the $16 \mathrm{Wr}$ group remained on the bar significantly increased from $28.6 \pm 11.8$ to $75.0 \pm 17.8 \mathrm{sec}(\mathrm{P}<0.05)$, as compared with $48 \mathrm{~h}$ after the final rotenone treatment. For rats in the $24 \mathrm{Wr}$ group, the time did not significantly increase between the 48-h and 2-week time periods ( $3.8 \pm 2.6$ and $6.3 \pm 5.9 \mathrm{sec}$, respectively) (Fig. 2B). This result suggested that the loss in motor ability could be recovered for $16 \mathrm{Wr}$, and may be predominantly affected by rotenone treatment; however, as motor ability could not recovered for $24 \mathrm{Wr}$, this may be caused by more serious organic damage. Furthermore, the weight and motor ability variation suggested that PD-associated features are progressively induced by rotenone treatment.

Rotenone treatment reduces TH-positive cell levels in the substantia nigra. The decrease of TH-positive cells in the substantia nigra is significant in patients with PD. As it was demonstrated that the behavior and motor ability of rats deteriorated after 16 weeks of rotenone treatment, the levels of TH-positive cells in the substantia nigra of these rats were measured and compared with those of $24 \mathrm{Wr}$ rats. As compared with the control group, TH-positive levels were significantly decreased in the $24 \mathrm{Wr}$ rats $(\mathrm{P}<0.05)$; however, no significant difference was detected in $16 \mathrm{Wr}$ rats (Fig. 3 ). This result suggested that, in $24 \mathrm{Wr}$ rats, the DA neurons in the substantia nigra were injured, whereas this damage was not as severe in $16 \mathrm{Wr}$ rats.

Rotenone treatment affects ATP, lactic acid, ND1 mRNA and LDHB mRNA levels in SM and VM. In the present study, the rotenone group developed parkinsonism, characterized by weight loss, loss of motor ability and a reduction in TH-positive cells in the substantia nigra, which was more severe in $24 \mathrm{Wr}$ rats, as compared with $16 \mathrm{Wr}$ rats. In order to investigate the development of accompanied energy stress, the levels of ATP, lactic acid, ND1 mRNA and LDHB mRNA were detected in SM and VM tissue samples. These targets are important in metabolism compensation and, like DA neurons are abundant in VM, SM is also a tissue with high energy consumption $(18,19)$. In order to elucidate the effect of rotenone treatment on the energy status of rats, the levels of 
A

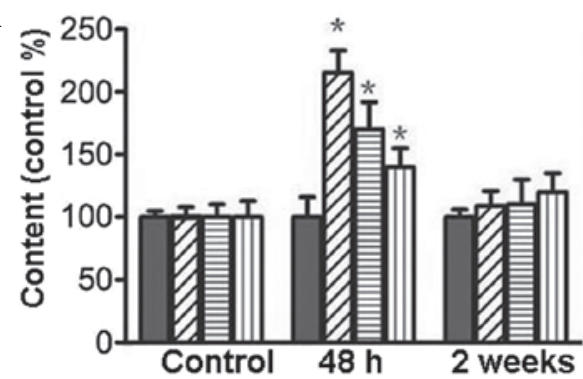

C

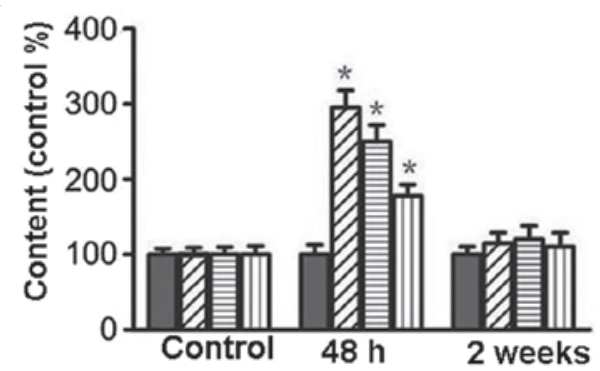

B

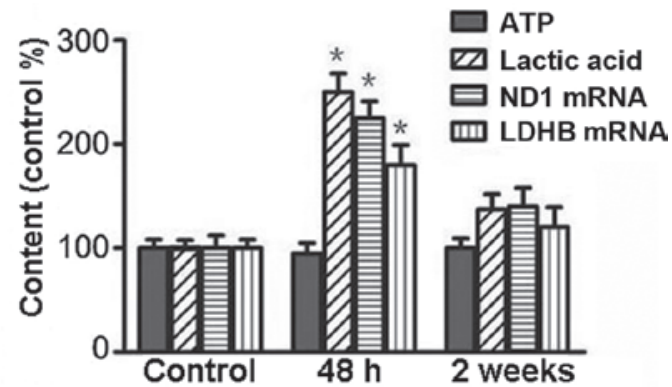

D

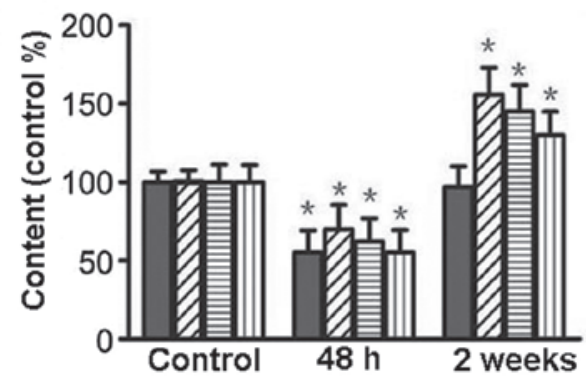

Figure 4. ATP, lactic acid, ND1 mRNA and LDHB mRNA levels in SM and VM tissues (n=3) in the control group and $48 \mathrm{~h}$ and 2 weeks after final rotenone treatment, respectively. (A) SM of rats that were treated with rotenone for 16 and (B) 24 weeks, respectively. (C) VM of rats that were treated with rotenone for 16 and (D) 24 weeks, respectively. Data are presented as the mean \pm standard deviation. "P<0.05 vs. the control group. ND1, NADH dehydrogenase 1; LDHB, lactate dehydrogenase B; SM, skeletal muscle; VM, ventral mesocerebrum.
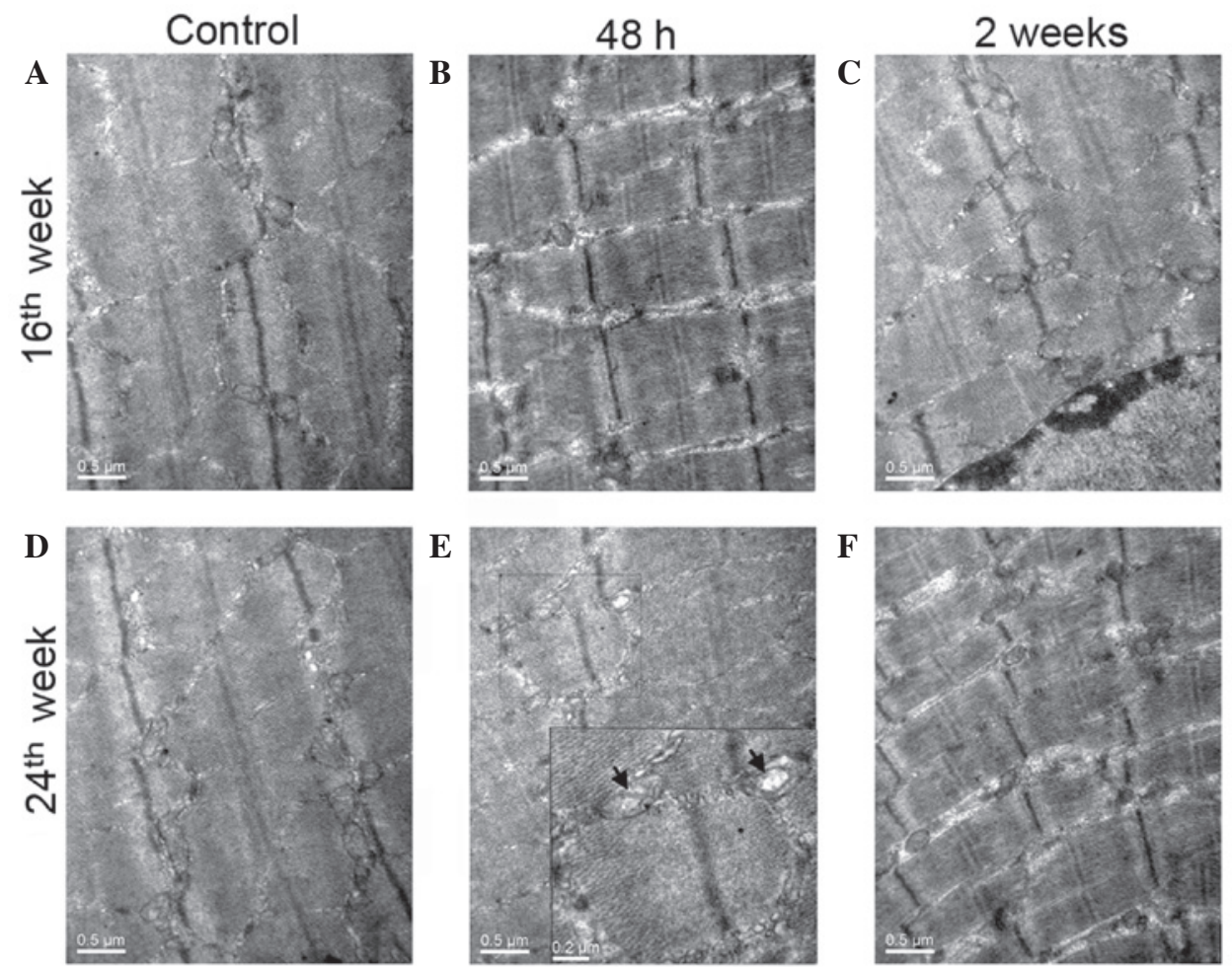

Figure 5. Representative electron microscope images of skeletal muscle (magnification, x25,000) in the (A and D) control group and the rotenone-treated groups (B and E) $48 \mathrm{~h}$ and (C and F) 2 weeks after the final rotenone treatment. Swollen mitochondria were detected, as indicated by the arrow (magnification, $\mathrm{x} 50,000$ ).

these targets were detected $48 \mathrm{~h}$ and 2 weeks after the final rotenone treatment in $16 \mathrm{Wr}$ and $24 \mathrm{Wr}$ rats.

The results demonstrated that ATP levels in SM were not affected by rotenone treatment, as no significant differences were detected between the rotenone and control groups
$48 \mathrm{~h}$ and 2 weeks after the final rotenone treatment in $16 \mathrm{Wr}$ (Fig. 4A) and 24Wr (Fig. 4B) rats. However, the effect of rotenone treatment on ATP levels in VM differed between the $16 \mathrm{Wr}$ (Fig. 4C) and $24 \mathrm{Wr}$ (Fig. 4D) rats. In $16 \mathrm{Wr}$ rats, ATP levels at $48 \mathrm{~h}$ and 2 weeks post-treatment were consistent 
with the control; however, in 24Wr rats, ATP levels significantly decreased $48 \mathrm{~h}$ after treatment $(55 \pm 14 \%$ of control; $\mathrm{P}<0.05)$, and subsequently recovered to the control level 2 weeks later.

The response of lactic acid, ND1 mRNA and LDHB mRNA levels to rotenone treatment was similar, and differences were detected between SM and VM tissues. In SM, the levels of all these targets significantly increased $(\mathrm{P}<0.05) 48 \mathrm{~h}$ after treatment and subsequently recovered to the control level 2 weeks later in $16 \mathrm{Wr}$ (Fig. 4A) and 24Wr (Fig. 4B) rats. As compared with the control, the respective levels of lactic acid, ND1 mRNA and LDHB mRNA in SM were $215 \pm 18,172 \pm 22$ and $143 \pm 15 \%$ in $16 \mathrm{Wr}$ rats, and $251 \pm 14,230 \pm 14$ and $184 \pm 19 \%$ in $24 \mathrm{Wr} 48 \mathrm{~h}$ after rotenone treatment. However, in VM tissue these levels differed between 16Wr (Fig. 4C) and 24Wr (Fig. 4D) rats. When measured $48 \mathrm{~h}$ after treatment, in $16 \mathrm{Wr}$ rats, these levels all significantly increased $(291 \pm 23,253 \pm 22$ and $180 \pm 15 \%$ of control, respectively; $\mathrm{P}<0.05)$; however, in $24 \mathrm{Wr}$ rats, these levels all significantly decreased $(71 \pm 15,62 \pm 15$ and $55 \pm 14 \%$ of control, respectively; $\mathrm{P}<0.05)$, as compared with the control. Testing 2 weeks later in $16 \mathrm{Wr}$ rats demonstrated that these levels recovered to the control level; whereas these levels remained significantly increased in $24 \mathrm{Wr}$ rats, as compared with the control $(156 \pm 17,150 \pm 16$ and $142 \pm 15 \%$ of control, respectively; $\mathrm{P}<0.05)$.

With energy stress, cells respond by enhancing their energy metabolism activity, therefore the levels of lactic acid, ND1 mRNA and LDHB mRNA increase; however, if energy stress is severe and the cells maximum energy producing capabilities are insufficient, cell dysfunction occurs with subsequent inhibition of energy producing activity $(20,21)$. These results suggested that, in VM tissue following rotenone treatment for 24 weeks, the energy producing capability of cells may have been attenuated, thus the energy disturbance induced by rotenone treatment could not be withstood. In sum, these findings suggested that the rotenone-induced damage to energy producing capabilities is selected, being more severe in VM, compared with SM.

Morphology of SM mitochondria affected by rotenone treatment. Analysis of ATP, lactic acid, ND1 mRNA and LDHB mRNA levels suggested that energy stress in $24 \mathrm{Wr}$ rats was compensated in SM; however, this was not demonstrated in VM tissue. In order to elucidate how rotenone treatment affects the energy metabolism ability of SM, the morphology of SM mitochondria were observed by electron microscope. The results demonstrated that the mitochondria of $24 \mathrm{Wr}$ rats were swollen $48 \mathrm{~h}$ post-treatment, however this was not observed in $16 \mathrm{Wr}$ rats, as compared with the control (Fig. 5). Mitochondrial swelling recovered after 2 weeks in $16 \mathrm{Wr}$ and $24 \mathrm{Wr}$ rats. Mitochondrial swelling is an indicator of the cell's compensatory response to energy stress. Therefore, this result suggested that, in SM, rotenone treatment induced more energy stress in $24 \mathrm{Wr}$ rats than in $16 \mathrm{Wr}$, which suggested that rotenone treatment weakened the energy producing capability of SM, although this effect was not as profound as the effect induced in VM.

\section{Discussion}

In the present study, rats were administered $1.0 \mathrm{mg} / \mathrm{kg}$ rotenone (i.p.) every $48 \mathrm{~h}$ which has been demonstrated to induce rotenone concentration fluctuation $(17,22)$, resulting in the repeated induction of energy stress in the rats. Energy stress has been demonstrated to burden mitochondria via energy metabolism compensation, which favors the production of reactive oxygen species $(23,24)$. Furthermore, energy stress may induce dysfunction in energy production and anti-oxidation systems $(20,21)$, which exacerbates the energy crisis and further damages the cells, particularly during energy decompensation. Therefore, in some energy sensitive cells, repeated energy stress may damage the energy producing ability of cells, which may accumulate and result in the cells being more sensitive to energy disturbances. Consistent with this hypothesis, the rats in the present study exhibited a progressed acute response and energy stress to rotenone treatment. In this way, the repeated rotenone treatment induced repeated energy stresses, which progressively decayed the cells energy production ability and made them more sensitive to further energy stress. It has previously been reported that systemic administration of rotenone in rats induced PD-associated pathology in chronic animal models, but not in the more energy disturbed acute models $(11,25,26)$. The lighter and sustained energy disturbance induced in the chronic model may have favored damage to the energy producing ability of energy sensitive DA cells and accumulated in them; whereas in the acute model, the severe energy disturbance was beyond the compensation ability of the tissues, therefore selective damage of DA cells was not induced. Therefore, future research into how the fragile cells were regulated to confront the energy stress may aid the elucidation of the underlying mechanisms of PD, which would build upon previous studies that have indicated PD-associated genes, parkin, phosphatase and tensin homolog-induced novel kinase-1 and DJ-1, are involved in energy crisis (27-29).

In the present study, an acute response was induced in the rotenone group following low dose rotenone treatment, which has seldom been reported by other researchers, which may be due to the use of senile rats in the present study. In the aged organisms, oxidative damage may accumulate more readily, leading to weakened energy producing capabilities (20), which favors the induction of energy crisis by rotenone treatment.

PD symptoms in patients are often acutely exacerbated by infection, inflammation, cold, fever and mental stress (30-33). With decreased energy producing capability, these situations may induce energy disturbance and subsequent energy crisis, which would exacerbated their PD symptoms and cause further damage to the sensitive DA cells. Further research is required to investigate how and to what extent factors such as fever, infection, cold, inflammation cytokine and environmental toxicants selectively disturb the energy metabolism of fragile cells and favor the progress of PD.

In conclusion, energy stress and parkinsonisms were induced and progressed following repeated rotenone treatment in rats in the present study. Notably, energy stress was more developed in VM, as compared with SM. The repeated energy stress demonstrated in the rats of the present study may be important for PD-associated selective injuries. Currently, there is no effective treatment for PD; therefore encouraging patients with PD to avoid any kind of energy disturbance and energy stress, and developing a therapeutic strategy that promotes the energy producing ability of cells may help to prevent and attenuate the progress of this disease. The results 
of the present study provided an understanding of the development of PD-associated energy stress, which may provide insight into PD pathogenesis and facilitate the exploration of novel therapeutic targets.

\section{Acknowledgements}

The present study was supported by the National Nature Science Foundation of China (grant no. 81370403).

\section{References}

1. Coelho M and Ferreira JJ: Late-stage Parkinson disease. Nat Rev Neurol 8: 435-442, 2012.

2. Kim YS, Kim YK, Hwang O and Kim DJ: Pathology of neurodegenerative diseases: InTech 2012: 99-138, 2012.

3. Gorell JM, Peterson EL, Rybicki BA and Johnson CC: Multiple risk factors for Parkinson's disease. J Neurol Sci 217: 169-174, 2004.

4. Kamel F, Tanner C, Umbach D, Hoppin J, Alavanja M, Blair A, Comyns K, Goldman S, Korell M and Langston J: Pesticide exposure and self-reported Parkinson's disease in the agricultural health study. Am J Epidemiol 165: 364-374, 2007.

5. Dunn L, Allen GF, Mamais A, Ling H, Li A, Duberley KE, Hargreaves IP, Pope S, Holton JL, Lees A, et al: Dysregulation of glucose metabolism is an early event in sporadic Parkinson's disease. Neurobiol Aging 35: 1111-1115, 2014.

6. Schapira AH: Mitochondria in the aetiology and pathogenesis of Parkinson's disease. Lancet Neurol 7: 97-109, 2008.

7. Ambrosi G, Ghezzi C, Sepe S, Milanese C, Payan-Gomez C, Bombardieri CR, Armentero MT, Zangaglia R, Pacchetti C, Mastroberardino PG and Blandini F: Bioenergetic and proteolytic defects in fibroblasts from patients with sporadic Parkinson's disease. Biochim Biophys Acta 1842: 1385-1394, 2014.

8. Bolam JP and Pissadaki EK: Living on the edge with too many mouths to feed: Why dopamine neurons die. Mov Disord 27: 1478-1483, 2012.

9. Panov A, Dikalov S, Shalbuyeva N, Taylor G, Sherer T and Greenamyre JT: Rotenone model of Parkinson disease: Multiple brain mitochondria dysfunctions after short term systemic rotenone intoxication. J Biol Chem 280: 42026-42035, 2005.

10. Sian J, Dexter DT, Lees AJ, Daniel S, Agid Y, Javoy-Agid F, Jenner P and Marsden CD: Alterations in glutathione levels in Parkinson's disease and other neurodegenerative disorders affecting basal ganglia. Ann Neurol 36: 348-355, 1994.

11. Greenamyre JT, Cannon JR, Drolet R and Mastroberardino PG: Lessons from the rotenone model of Parkinson's disease. Trends Pharmacol Sci 31: 141-142, 2010.

12. Livak KJ and Schmittgen TD: Analysis of relative gene expression data using real-time quantitative PCR and the $2^{-\Delta \Delta C}$ method. Methods 25: 402-408, 2001.

13. Greene JG, Noorian AR and Srinivasan S: Delayed gastric emptying and enteric nervous system dysfunction in the rotenone model of Parkinson's disease. Exp Neurol 218: 154-161, 2009.

14. Drolet RE, Cannon JR, Montero L and Greenamyre JT: Chronic rotenone exposure reproduces Parkinson's disease gastrointestinal neuropathology. Neurobiol Dis 36: 96-102, 2009.

15. Cersosimo MG and Benarroch EE: Pathological correlates of gastrointestinal dysfunction in Parkinson's disease. Neurobiol Dis 46: 559-564, 2012.
16. Abbott RA, Cox M, Markus $\mathrm{H}$ and Tomkins A: Diet, body size and micronutrient status in Parkinson's disease. Eur J Clin Nutr 46: 879-884, 1992.

17. Cannon JR, Tapias V, Na HM, Honick AS, Drolet RE and Greenamyre JT: A highly reproducible rotenone model of Parkinson's disease. Neurobiol Dis 34: 279-290, 2009.

18. Greene JG, Dingledine R and Greenamyre JT: Neuron-selective changes in RNA transcripts related to energy metabolism in toxic models of parkinsonism in rodents. Neurobiol Dis 38: 476-481, 2010.

19. Crane, JD, Abadi A, Hettinga BP, Ogborn DI, MacNeil LG, Steinberg GR and Tarnopolsky MA: Elevated mitochondrial oxidative stress impairs metabolic adaptations to exercise in skeletal muscle. PLoS One 8: e81879, 2013.

20. Desler C, Hansen TL, Frederiksen JB, Marcker ML, Singh KK and Juel Rasmussen L: Is there a link between mitochondrial reserve respiratory capacity and aging? J Aging Res 2012: 192503, 2012.

21. Yap LP, Garcia JV, Han D and Cadenas E: The energy-redox axis in aging and age-related neurodegeneration. Adv Drug Deliv Rev 61: 1283-1298, 2009.

22. Caboni P, Sherer TB, Zhang N, Taylor G, Na HM, Greenamyre JT and Casida JE: Rotenone, deguelin, their metabolites, and the rat model of Parkinson's disease. Chem Res Toxicol 17: 1540-1548, 2004.

23. Guarente L, Partridge L and Wallace DC (eds): Molecular Biology of Aging. Cold Spring Harbor Laboratory Press, New York, NY, pp 1-20, 2007.

24. Fern R: Variations in spare electron transport chain capacity: The answer to an old riddle? J Neurosci Res 71: 759-762, 2003.

25. Blesa J, Phani S, Jackson-Lewis V and Przedborski S: Classic and new animal models of Parkinson's disease. J Biomed Biotechnol 2012: 845618, 2012.

26. Lapointe N, St-Hilaire M, Martinoli MG, Blanchet J, Gould P, Rouillard C and Cicchetti F: Rotenone induces non-specific central nervous system and systemic toxicity. FASEB J 18: 717-719, 2004.

27. Hilker R, Pilatus U, Eggers C, Hagenah J, Roggendorf J, Baudrexel S, Klein JC, Neumaier B, Fink GR, Steinmetz $\mathrm{H}$, et al: The bioenergetic status relates to dopamine neuron loss in familial PD with PINK1 mutations. PLoS One 7: e51308, 2012.

28. Jendrach M, Gispert S, Ricciardi F, Klinkenberg M, Schemm R and Auburger G: The mitochondrial kinase PINK1, stress response and Parkinson's disease. J Bioenerg Biomembr 41: 481-486, 2009.

29. Morais VA, Haddad D, Craessaerts K, De Bock PJ, Swerts J, Vilain S, Aerts L, Overbergh L, Grünewald A, Seibler P, et al: PINK1 loss-of-function mutations affect mitochondrial complex I activity via NdufA10 ubiquinone uncoupling. Science 344: 203-207, 2014.

30. Hashimoto T, Tokuda T, Hanyu N, Tabata K and Yanagisawa N: Withdrawal of levodopa and other risk factors for malignant syndrome in Parkinson's disease. Parkinsonism Relat Disord 9 (Suppl 1): S25-S30, 2003.

31. Pott Godoy MC, Tarelli R, Ferrari CC, Sarchi MI and Pitossi FJ: Central and systemic IL-1 exacerbates neurodegeneration and motor symptoms in a model of Parkinson's disease. Brain 131: 1880-1894, 2008.

32. Macht M, Brandstetter S and Ellgring $H$ : Stress affects hedonic responses but not reaching-grasping in Parkinson's disease. Behav Brain Res 177: 171-174, 2007.

33. Snyder AM, Stricker EM and Zigmond MJ: Stress-induced neurological impairments in an animal model of Parkinsonism. Ann Neurol 18: 544-551, 1985. 